\title{
A new selective fluorescent probe based on tamoxifen
}

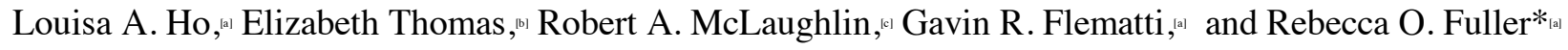

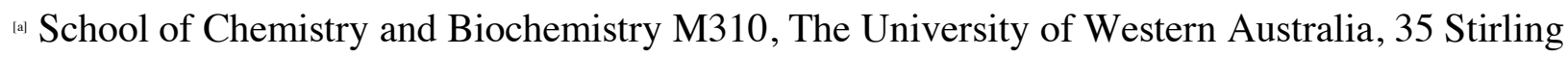
Hwy, Crawley WA 6009, Australia.

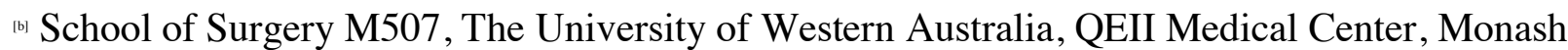
Ave, Nedlands, WA 6009, Australia.

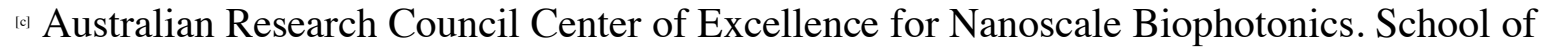
Medicine, University of Adelaide, Adelaide, SA 5005 Australia

Corresponding author:* Dr Rebecca Fuller

School of Chemistry and Biochemistry, M310

The University of Western Australia

35 Stirling Hwy

Crawley, WA 6009 Australia

Tel:+61-8-6488-4489

Fax:+61-8-6488-7247

E:rebecca.fuller@uwa.edu.au

GRAPHICAL ABSTRACT 


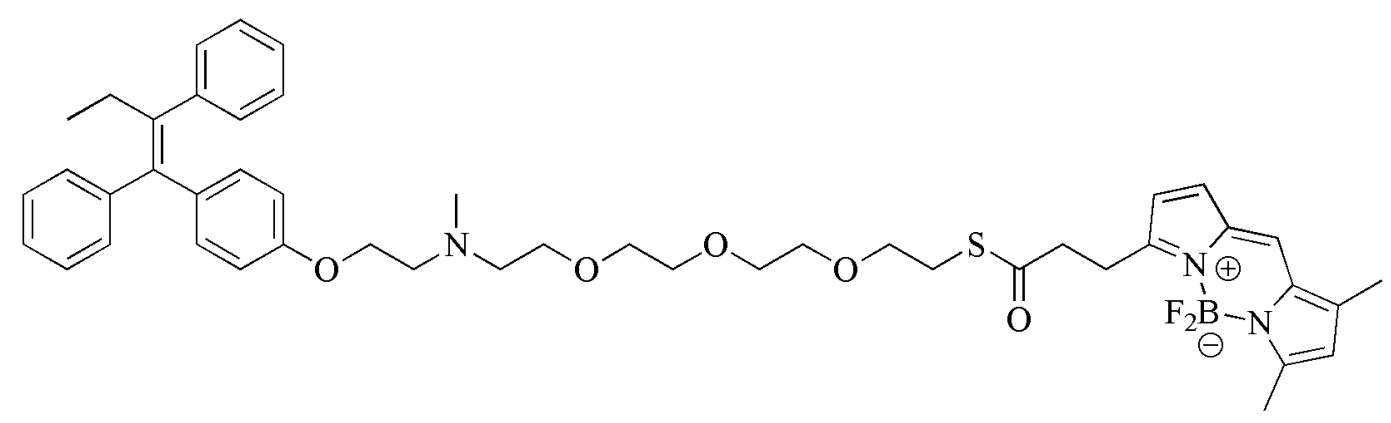

ABSTRACT

Developing targeted validation probes that can interrogate biology is of interest for both chemists and biologists. The synthesis of suitable compounds provides a means for avoiding the costly labeling of cells with specific antibodies and the bias associated with the interpretation of biological validation experiments. The chemotherapeutic agent, tamoxifen has been routinely used in the treatment of breast cancer for decades. Once metabolized, the active form of tamoxifen (4-hydroxytamoxifen) competes with the binding of estrogens to the estrogen receptors (ER). Its selectivity in ER modulation makes it an ideal candidate for the development of materials to be used as chemical probes. Here we report the synthesis of a fluorescent BODIPY®FL conjugate of tamoxifen linked through an ethylene glycol moiety, and present proof-of-principle results in ER positive and ER negative cell lines. Optical microscopy indicates that the fluorescent probe binds selectively to tamoxifen sensitive breast cancer cell lines. The compound showed no affinity for the tamoxifen resistant breast cancer lines. The specificity of the new compound make it a valuable addition to the chemical probe tool kit for estrogen receptors.

KEYWORDS Tamoxifen $•$ Fluorescent probe $\bullet$ fluorescent tamoxifen derivative $\bullet$ Estrogen Receptor 
Estrogens, such as $17 \beta$-estradiol, are well-known steroid hormones that play a central role in regulating a number of normal cell processes including proliferation and differentiation..$^{12}$ These hormones bind to a number of different receptors in cells, including the estrogen receptor (ER). The binding of the steroid to this nuclear receptor leads to a conformational change of the ER which results in the formation of a docking site for the binding of cofactors needed for transcription. ${ }^{3}$ Overexpression in the ER is associated with $75-80 \%$ of breast cancers. ${ }^{45}$ Hence ER ligands have found widespread clinical use in modern medicine. Tamoxifen (Figure 1) is one such example that is widely administered for the treatment of breast cancer. This non-steroidal small molecule modulates estrogen receptors (ER). ${ }^{6}$ By competing for binding with the $17 \beta-$ estradiol hormone it prevents the conformational change of the ER, and hence the associated transcription no longer occurs..$^{78}$

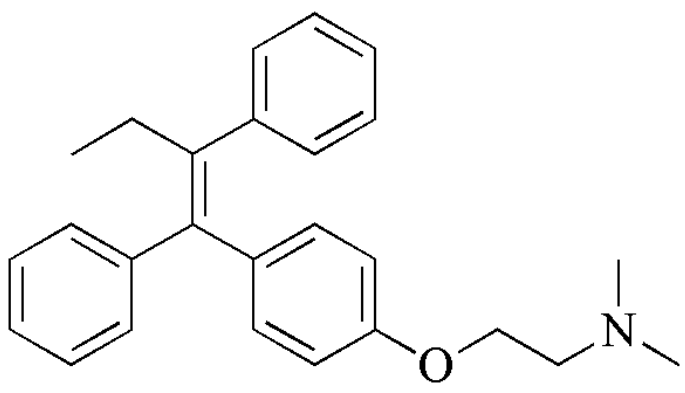

Figure 1 Tamoxifen

Despite the clinical success of Tamoxifen, like other estrogen therapies it has side effects for non-target tissues, including hot flushes as well as more serious conditions such as endometrial cancer. These adverse side affects are likely the result of the tamoxifen acting through different targets. ${ }^{5,0}$ It is believed that the endometrial cancer could be due to the formation of DNA adducts." However, the exact mechanisms are still unknown, which in part is due to a lack of experimental tools to identify targets. Fluorescently tagging bioactive molecules through the 
conjugation of a fluorophore to a bioactive compound is one solution to this problem. These compounds are known to provide an invaluable tool for cellular-based microscopy. ${ }^{2}$

The development of new chemical probes based on Tamoxifen is one strategy for further understanding the mechanisms of estrogen signaling and the specific physiological responses associated. Tamoxifen and the active metabolite 4-hydroxytamoxifen (OHT) represent ideal candidates to base new ER targeted compounds on. Despite the simplicity of this approach, relatively few examples are found for these compounds. ${ }^{1315}$ Although these probes would be useful in ER detection and imaging, ${ }^{14}$ they are unlikely to be useful for investigating the much debated membrane associated ERs. ${ }^{1617}$ This is because these probes either have no specificity for breast cancer cell lines, ${ }^{13}$ or do not involve the addition of a linker molecule, ${ }^{14.15}$ known to be a useful strategy for developing conjugates that target membrane receptors. ${ }^{18}$ The loss of specificity and complex uptake pattern seen by others, ${ }^{13}$ was attributed to several factors including the hydrophobic nature of the linker and/or fluorophore tags. ${ }^{19}$ In this work we have attempted to simplify the cellular localisation while maintaining selectivity of the tamoxifen probe through the introduction of a more hydrophilic linker shown to maintain ER selectivity ${ }^{20}$ We herein report the synthesis of a fluorescent analogue of tamoxifen, based on conjugating the BODIPY@FL fluorophore through an ethylene glycol moiety. We find that our new probe appears to be both cell permeable and selective.

Tamoxifen represents an ideal small molecule to base ER probes on. However, to date only three examples of fluorescent Tamoxifen probes have been developed. ${ }^{13-15}$ Two of these were based on the direct attachment of dyes to tamoxifen or its metabolite. ${ }^{14}$ Although these probes have good 
binding affinity and selectivity, they would not be useful for interpreting the contentious membrane receptor problem. To date the only tamoxifen probes developed for membrane associated ER studies have had poor affinity and loss of specificity compared to the parent compound..$^{13}$ The design of our conjugate has been in response to this.

Since the triphenylethylene core is the motif required to bind to the ER, the attachment of the dye has been made through the basic alkylaminoethoxy side chain, which is accepted to protrude out of the receptor binding pocket. ${ }^{21}$ A short ethylene glycol linker was thought to be the best approach for the conjugation of the dye to the tamoxifen. Bulky hydrophobic side chains have been proposed for reduced specificity and affinity in conjugated fluorescent tamoxifen. ${ }^{13}$ It has been predicted that the highest binding affinities are observed for bivalent 4-hydroxytamoxifen compounds with very short ethylene glycol linkers. ${ }^{2223}$ Cell specificity was achieved for an optical probe based on a nanoparticle conjugated to tamoxifen through a short ethylene glycol. ${ }^{20}$

Although the metabolite of the drug, OHT has 30-100 times more affinity for the ER,$_{, 4}$ we have chosen to tether the fluorophore directly to tamoxifen. In addition to being commercially available, sufficient binding has been found for probes based on tamoxifen rather than the metabolite..$^{5}$ A large number of commercial dyes exist, we have chosen to use the BODIPY ${ }^{\circ} F L$ fluorophore as it has been well characterized as a cell permeable fluorophore. Although not cell specific the BODIPY ${ }^{\circledR}$ FL conjugated OHT has displayed unusual localisation in the cytoplasm making it an ideal choice for our dye. ${ }^{19.13}$ 
The synthesis of tamoxifen conjugated with BODIPY®FL is outlined in Scheme 1. We have used a traditional conjugation approach to develop the probe compound based on tamoxifen..$^{25}$ Briefly, the tertiary amine of commercially available tamoxifen was demethylated using $\alpha$ chloroethyl chloroformate to produce the quaternary ammonium salt $1 .{ }^{26}$ Subsequent nucleophilic substitution between monotosylated polyethylene glycol $\mathbf{2}^{27}$ and the amine hydrochloride $\mathbf{1}$ afforded tertiary amine $\mathbf{3}$. The terminal hydroxyl group of $\mathbf{3}$ was then tosylated to give compound $\mathbf{4}$, and then substituted with thioacetate to give $\mathbf{5}$. Thioacetate $\mathbf{5}$ was then hydrolysed to give the thiol 6 and subsequently conjugated with the the thiol active maleimide BODIPY®FL fluorophore NHS ester to give the BODIPY®FL conjugated tamoxifen 7. 
(2)

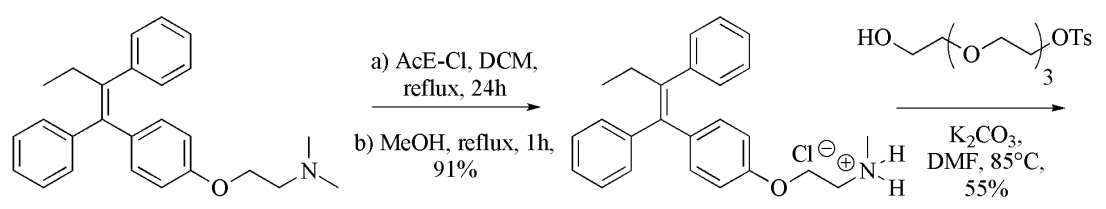

(1)

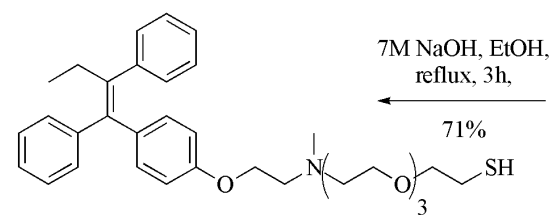

(6) BODIPY® FL NHS ester,
DMSO, rt, $24 \mathrm{~h}$

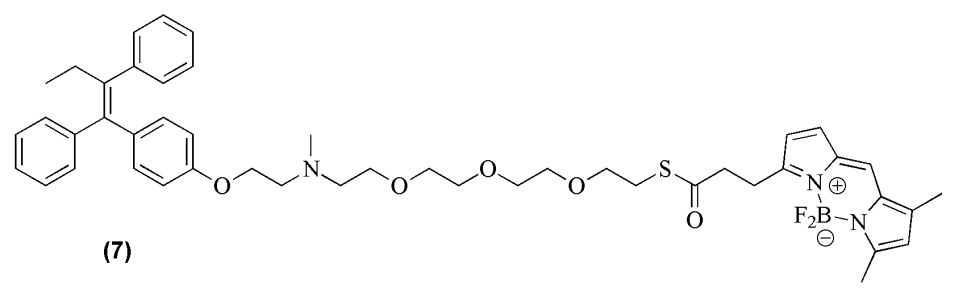

Scheme 1 Synthesis of BODIPY®FL conjugate of tamoxifen 7.

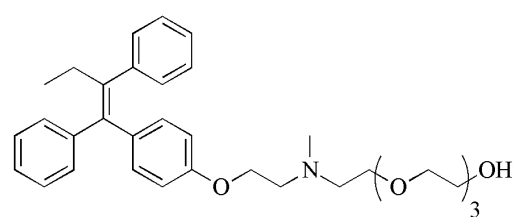

(3)

$$
\begin{gathered}
\mathrm{Ag}_{2} \mathrm{O}, \mathrm{KI}, \\
\mathrm{TsCl}, \mathrm{DCM}, \mathrm{rt}, \\
75 \%
\end{gathered}
$$

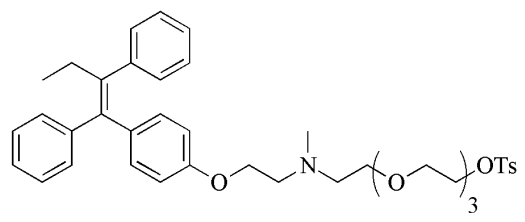

(4)

The cellular localisation of 7 in ER-positive MCF7 and ER-negative MDA231 breast cell lines was visualised by fluorescent confocal microscopy. ${ }^{28}$ Following incubation of the cells with the conjugate, uptake studies found that 7 was internalised in the ER-positive but not ER-negative cells (Figure 2). The specific uptake pattern suggests a receptor-mediated mechanism of uptake. No difference in degree of uptake or localisation is found with an increase in concentration of 7. We did not find any localisation of the BODIPY®FL conjugate in the cytoplasm. 


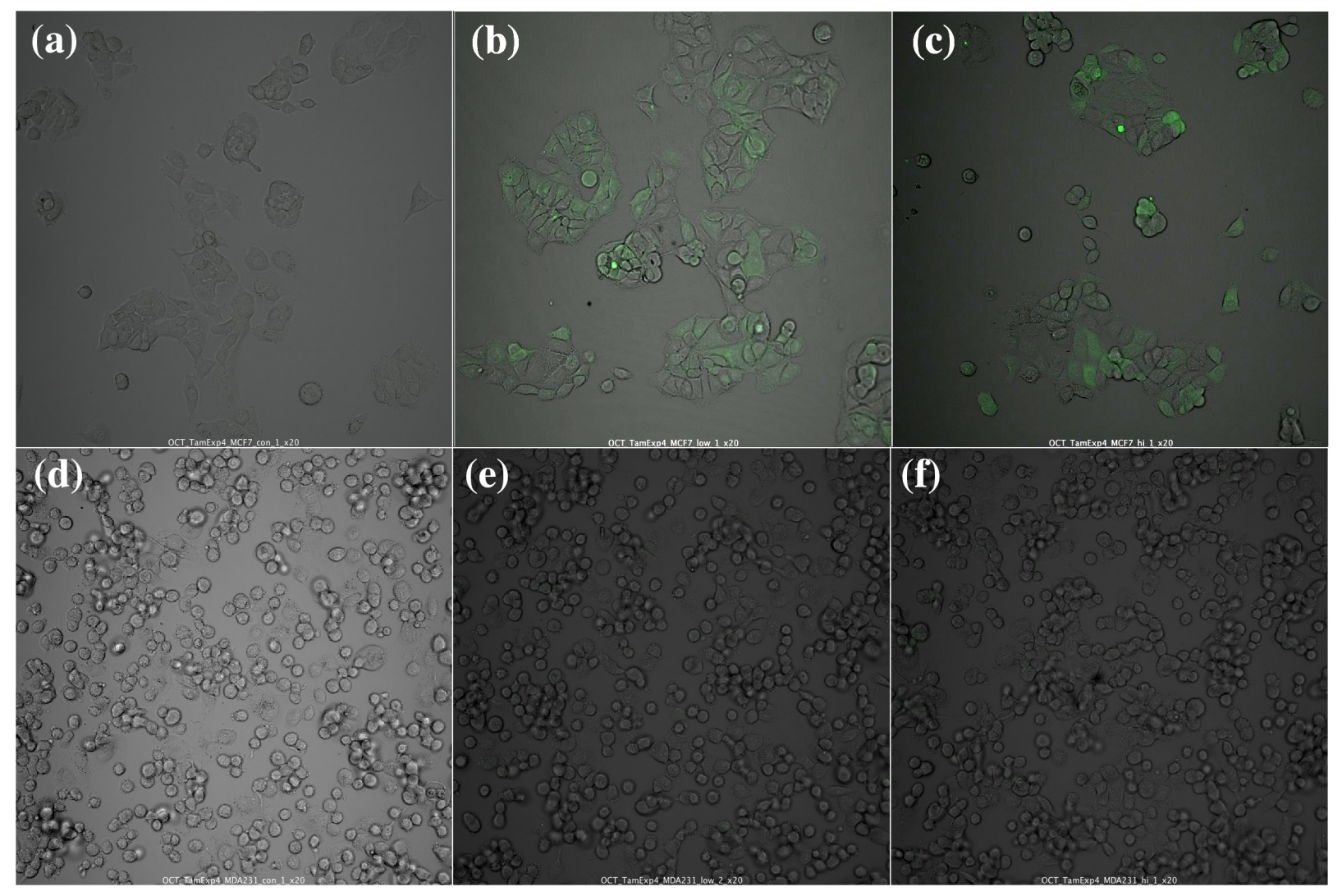

Figure 2. Confocal images of MCF7 (a-c) and MDA231 (d-f) cells following incubation with low (b,e) and high (c,f) concentrations of (7) compared to untreated control cells (a) and (d).

Tamoxifen has been administered as a chemotherapeutic agent for the treatment of breast cancer for over three decades. The active form of the drug competes with estrogen in the binding of ER sites. The selectivity of this small compound makes it an ideal candidate to develop fluorescentbased detection tools. Despite the potential of this small compound there are very few examples of fluorescent-based analogues found in the literature, and those that have been reported have associated drawbacks. Hence, this project has involved the development of a fluorescently labeled analogue of tamoxifen by conjugating a fluorescent BODIPY®FL group via an ethylene glycol linker unit. Preliminary cell testing shows that the molecule has maintained its cell 
specificity in breast lines and lays the foundation for further characterization with other estrogen receptor positive cell lines.

\section{Acknowledgements}

Dr Anthony Reeder (CMCA-UWA) for help with mass spectra acquisition. ROF would like to acknowledge financial support from Cancer Council Western Australia through a Susan Cavanagh ECI grant, the Ada Bartholomew Medical Research Trust, Breast Cancer Research Center WA, and The University of Western Australia ReEntry Fellowship. RAM would like to acknowledge financial support from the Australian Research Council and the National Health and Medical Research Council, Australia.

\section{References and notes}

1. Avendaño, C.; Menéndez, J. C. In Medicinal Chemistry of Anticancer Drugs; Elsevier: Amsterdam, 2008; pp. 53-91.

2. Weatherman, R. V. In Nuclear Receptors as Drug Targets; Wiley-VCH Verlag GmbH \& Co. KGaA, 2008; pp. 47-64.

3. Mangelsdorf, D. J.; Thummel, C.; Beato, M.; Herrlich, P.; Schütz, G.; Umesono, K.; Blumberg, B.; Kastner, P.; Mark, M.; Chambon, P.; Evans, R. M. Cell 1995, 83, 835-839.

4. $\quad$ Cleator, S.; Heller, W.; Coombes, R. C. Lancet Oncol. 2007, 8, 235-244.

5. Dahlman-Wright, K.; Cavailles, V.; Fuqua, S. A.; Jordan, V. C.; Katzenellenbogen, J. A.; Korach, K. S.; Maggi, A.; Muramatsu, M.; Parker, M. G.; Gustafsson, J.-Å. Pharmacol. Rev. 2006, 58, 773-781.

6. O'Regan, R. M.; Jordan, V. C. Lancet Oncol. 2002, 3, 207-214.

7. Jordan, V. C. Nat Rev Cancer 2007, 7, 46-53.

8. Jordan, V. C. Nat Rev Drug Discov 2003, 2, 205-213. 
9. Deligdisch, L. Modern pathology : an official journal of the United States and Canadian Academy of Pathology, Inc 1993, 6, 94-106.

10. Díaz, M.; Marrero-Alonso, J.; Marrero, B. G.; Marín, R.; Gomez, T.; Alonso, R. In Selective Estrogen Receptor Modulators : A New Brand of Multitarget Drugs; Cano, A.; Calaf i Alsina, J.; Duenas-Diez, J. L. Eds.; Springer-Verlag Berlin Heidelberg, 2006; pp. 79-96.

11. Hard, G. C.; Iatropoulos, M. J.; Jordan, K.; Radi, L.; Kaltenberg, O. P.; Imondi, A. R.; Williams, G. M. Cancer Res. 1993, 53, 4534-4541.

12. Bunnage, M. E.; Piatnitski, E. L.; Jones, C.; Jones, L. H. Nat. Chem. Biol. 2013, 9, 195199.

13. Rickert, E. L.; Oriana, S.; Hartman-Frey, C.; Long, X.; Webb, T. T.; Nephew, K. P.; Weatherman, R. V. Bioconjugate Chem. 2010, 21, 903-910.

14. Abendroth, F.; Solleder, M.; Mangoldt, D.; Welker, P.; Licha, K.; Weber, M.; Seitz, O. Eur. J. Org. Chem. 2015, 2015, 2157-2166.

15. Marrero-Alonso, J.; Morales, A.; García Marrero, B.; Boto, A.; Marín, R.; Cury, D.; Gómez, T.; Fernández-Pérez, L.; Lahoz, F.; Díaz, M. Eur. J. Pharm. Biopharm. 2013, 85, 898-910.

16. Pietras, R. J.; Szego, C. M. Nature 1977, 265, 69-72.

17. Govind, A. P.; Thampan, R. V. Mol. Cell. Biochem. 2003, 233, 233-240.

18. Revankar, C. M.; Mitchell, H. D.; Field, A. S.; Burai, R.; Corona, C.; Ramesh, C.; Sklar, L. A.; Arterburn, J. B.; Prossnitz, E. R. ACS Chem. Biol. 2007, 2, 536-544.

19. Yu, B.; Qin, Z.; Wijewickrama, G. T.; Edirisinghe, P.; Bolton, J. L.; Thatcher, G. R. J. Bioconjugate Chem. 2009, 20, 728-741.

20. Dreaden, E. C.; Mwakwari, S. C.; Sodji, Q. H.; Oyelere, A. K.; El-Sayed, M. A. Bioconjugate Chem. 2009, 20, 2247-2253.

21. Shiau, A. K.; Barstad, D.; Loria, P. M.; Cheng, L.; Kushner, P. J.; Agard, D. A.; Greene, G. L. Cell 1998, 95, 927-937.

22. Shan, M.; Carlson, K. E.; Bujotzek, A.; Wellner, A.; Gust, R.; Weber, M.; Katzenellenbogen, J. A.; Haag, R. ACS Chem. Biol. 2013, 8, 707-715.

23. Shan, M.; Bujotzek, A.; Abendroth, F.; Wellner, A.; Gust, R.; Seitz, O.; Weber, M.; Haag, R. ChemBioChem 2011, 12, 2587-2598.

24. Desta, Z.; Ward, B. A.; Soukhova, N. V.; Flockhart, D. A. J. Pharmacol. Exp. Ther. 2004, 310, 1062-1075.

25. General experimental: All reactions were carried out under an argon gas atmosphere in flame-dried glassware with magnetic stirring, unless otherwise stated. All reactions involving heating were placed into a preheated oil bath at the specified temperature, unless otherwise stated. Solvents were used dry, unless otherwise stated. Solvents were dried and purified according to the methods described by Armarego W. L. K. and Chai C. In Purification of Laboratory Chemicals $5^{\text {th }}$ Ed; Butterworth-Heinememann: Cornwall, 2003. Solvents used for preparative HPLC were HPLC grade acetonitrile and Milli-Q water obtained by filtration of deionised (DI) water through a Milli-Q ultrapure water sytem (Millipore, Australia). All reagents were purchased from Sigma-Aldrich, Fluka, Merck, or Boron Molecular and used without further purification, unless otherwise stated.

Thin layer chromatography (TLC) was performed on Merck silica gel $60 \mathrm{~F}_{254}$ pre-coated aluminium sheets. Visualisation of developed plates was achieved through the use of a $254 \mathrm{~nm}$ or 
$365 \mathrm{~nm}$ UV lamp or staining with phosphomolybdic acid stain solution. Column chromatography was performed using silica gel $60(0.063-0.200 \mathrm{~nm})$ as supplied by Merck, unless otherwise stated. HPLC was conducted using an Agilent 1200 with a photodiode array detector (PDA). Separation was achieved using a $250 \times 10 \mathrm{~mm}$ i.d., $5 \mu \mathrm{m}$, Apollo $\mathrm{C}_{18}$ reversed phase column (Grace-Division) with a $33 \mathrm{~mm} \times 7 \mathrm{~mm}$ guard column of the same material.

${ }^{1} \mathrm{H}$ and ${ }^{13} \mathrm{C}$ NMR spectra were acquired in the specified deuterated solvent using either a Bruker AV600 (600.13 MHz for ${ }^{~} \mathrm{H}$ and $150.9 \mathrm{MHz}$ for $\left.{ }^{ } \mathrm{C}\right)$, a Bruker AV500 (500.13 MHz for $\mathrm{H}$ and 125.8 MHz for ${ }^{13} \mathrm{C}$ ), or a Varian Gemini-400 $\left(399.85 \mathrm{MHz}\right.$ for ${ }^{1} \mathrm{H}$ and $100.5 \mathrm{MHz}$ for ${ }^{13} \mathrm{C}$ ) spectrometer at $25^{\circ} \mathrm{C}$. Chemical shifts are reported in parts per million downfield from tetramethylsilane using the residual solvent resonance as internal standard. ${ }^{201}$ Data are reported as follows: chemical shift, multiplicity (app = apparent, $\mathrm{s}=$ singlet, $\mathrm{d}=$ doublet, $\mathrm{t}=$ triplet, $\mathrm{q}=$ quartet, $\mathrm{m}=$ multiplet, $\mathrm{br}=$ broad, sept = septet), coupling constant, integration, and assignment. High resolution mass spectra (HRMS) were acquired on a Waters liquid chromatograph premier (LCT) mass spectrometer using electrospray ionisation (ESI).

Synthesis of N-Desmethyl Tamoxifen (1): Tamoxifen $(2.12 \mathrm{~g}, 5.71 \mathrm{mmol})$ was dissolved in anhydrous $\mathrm{CH}_{2} \mathrm{Cl}_{2}(60 \mathrm{~mL})$ and the mixture was cooled to $0^{\circ} \mathrm{C}$ in an ice bath, under an atmosphere of argon gas. To this cooled mixture was added 1-chloroethyl chloroformate $(0.68$ $\mathrm{mL}, 0.90 \mathrm{~g}, 6.30 \mathrm{mmol}$ ) in one portion. After $15 \mathrm{~min}$ stirring at $0^{\circ} \mathrm{C}$, the resulting mixture was heated at reflux for $22 \mathrm{hr}$. After this time, the solvent was removed under reduced pressure to obtain a yellow oil. The crude mixture was then dissolved in methanol, refluxed for a further 3 hours, and then concentrated under reduced pressure. The resulting crude material was subjected to silica gel flash column chromatography $\left(\mathrm{CH}_{2} \mathrm{Cl}_{2} \rightarrow 10: 90 \mathrm{MeOH} / \mathrm{CH}_{2} \mathrm{Cl}_{2}\right)$ to give compound 1 as a colourless solid $\left(2.05 \mathrm{~g}, 91 \%\right.$ yield, $R_{f}=0.46$ in $\left.1: 10 \mathrm{MeOH} / \mathrm{CH}_{2} \mathrm{Cl}_{2}\right)$. ${ }^{\mathbf{H}} \mathbf{N M R}(399.8 \mathrm{MHz}$, $\mathrm{d}_{6}$-DMSO): $\delta=7.38-7.30(\mathrm{~m}, 2 \mathrm{H}, 2 \times \mathrm{Ar} \underline{\mathrm{H}}), 7.26-7.05(\mathrm{~m}, 8 \mathrm{H}, 8 \times \mathrm{Ar} \underline{\mathrm{H}}), 6.76-6.69(\mathrm{~m}, 2 \mathrm{H}, 2 \times$ $\operatorname{Ar} \underline{\mathrm{H}}), 6.66-6.58(\mathrm{~m}, 2 \mathrm{H}, 2 \times \mathrm{Ar} \underline{\mathrm{H}}), 4.06(\mathrm{t}, J=5.2 \mathrm{~Hz}, 2 \mathrm{H}), 3.16(\mathrm{t}, J=5.2 \mathrm{~Hz}, 2 \mathrm{H}), 2.32(\mathrm{q}, J=$ $\left.7.2 \mathrm{~Hz}, 2 \mathrm{H}, \mathrm{C}_{2} \mathrm{CH}_{3}\right), 0.80\left(\mathrm{t}, J=7.2 \mathrm{~Hz}, 3 \mathrm{H}, \mathrm{CH}_{2} \underline{\mathrm{CH}}_{3}\right) ;{ }^{1} \mathbf{C} \mathbf{N M R}\left(125.77 \mathrm{MHz}, \mathrm{CD}_{3} \mathrm{OD}\right): \delta=$

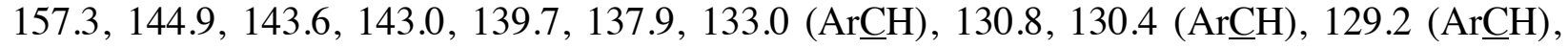
$128.9(\mathrm{Ar} \underline{\mathrm{CH}}), 127.7(\mathrm{Ar} \underline{\mathrm{CH}}), 127.2(\mathrm{Ar} \underline{\mathrm{CH}}), 114.6(\mathrm{Ar} \underline{\mathrm{CH}}), 64.2\left(\underline{\mathrm{CH}}_{2}\right), 49.5\left(\underline{\mathrm{CH}}_{2}\right), 33.8\left(\underline{\mathrm{C}} \mathrm{H}_{3}\right)$, $29.9\left(\underline{\mathrm{CH}}_{2}\right), 13.8\left(\underline{\mathrm{CH}}_{3}\right)$; HRMS (ESI): calc. Chemical Formula: $\mathrm{C}_{25} \mathrm{H}_{28} \mathrm{NO}[\mathrm{M}+\mathrm{H}-\mathrm{Cl}]+358.2171$, found 358.2162 .

Synthesis of 2-(2-(2-(2-hydroxyethoxy)ethoxy)ethoxy)ethyl 4-methylbenzenesulfonate (2): Tetraethylene glycol (2 mL, $2.25 \mathrm{~g}, 11.58 \mathrm{mmol})$ was dissolved in anhydrous $\mathrm{CH}_{2} \mathrm{Cl}_{2}(25 \mathrm{~mL})$ and the mixture was cooled to $0^{\circ} \mathrm{C}$ in an ice bath, under an atmosphere of argon gas. To this cooled mixture was added freshly prepared $\mathrm{Ag}_{2} \mathrm{O}(4.03 \mathrm{~g}, 17.39 \mathrm{mmol}), \mathrm{KI}(0.77 \mathrm{~g}, 4.64 \mathrm{mmol})$, and $\mathrm{TsCl}(2.21 \mathrm{~g}, 11.59)$. The resulting mixture was left to stir at $0^{\circ} \mathrm{C}$ for $30 \mathrm{~min}$. After this time, the $\mathrm{Ag}_{2} \mathrm{O}$ was filtered off through a pad of celite eluted with $1: 12 \mathrm{MeOH} / \mathrm{CH}_{2} \mathrm{Cl}_{2}$. The filtrate was then concentrated under reduced pressure. The resulting crude material was subjected to silica gel column chromatography $\left(\mathrm{CH}_{2} \mathrm{Cl}_{2} \rightarrow 1: 4\right.$ acetone $\left./ \mathrm{CH}_{2} \mathrm{Cl}_{2}\right)$ to give compound $\mathbf{2}$ as a colourless oil $\left(1.78 \mathrm{~g}, 44 \%\right.$ yield, $\mathrm{R}_{\mathrm{f}}=0.58$ in $1: 8$ acetone $\left./ \mathrm{CH}_{2} \mathrm{Cl}_{2}\right) .{ }^{1} \mathbf{H} \mathbf{N M R}\left(399.85 \mathrm{MHz}, \mathrm{CDCl}_{3}\right): \delta=7.78$ $(\operatorname{app~d}, \mathrm{J}=8.4 \mathrm{~Hz}, 2 \mathrm{H}, 2 \times \operatorname{Ar} \underline{\mathrm{H}}), 7.34(\operatorname{app~d}, \mathrm{J}=8.4 \mathrm{~Hz}, 2 \mathrm{H}, 2 \times \operatorname{Ar} \underline{\mathrm{H}}), 4.16-4.13(\mathrm{~m}, 2 \mathrm{H}$, $\left.\mathrm{CH}_{2} \mathrm{OTs}\right), 3.70-3.56\left(\mathrm{~m}, 14 \mathrm{H}, 7 \times \mathrm{OC}_{2}\right), 2.93(\mathrm{~s}, 1 \mathrm{H}, \mathrm{OH}), 2.43\left(\mathrm{~s}, 3 \mathrm{H}, \underline{\mathrm{CH}}_{3}\right) ;{ }^{13} \mathbf{C ~ N M R}(100.54$

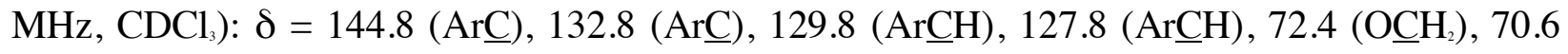




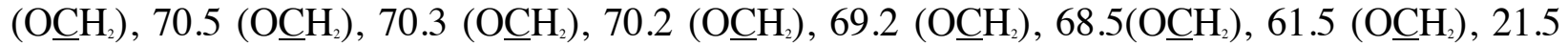
$\left(\underline{\mathrm{CH}}_{3}\right) ; \mathrm{HRMS}$ (ESI): calc. $\mathrm{C}_{15} \mathrm{H}_{25} \mathrm{O}_{7} \mathrm{~S}[\mathrm{M}+\mathrm{H}]+349.1321$, found 349.1323 .

Synthesis of (E)-1-(4-(1,2-diphenylbut-1-en-1-yl)phenoxy)-3-methyl-6,9,12-trioxa-3azatetradecan-14-ol (3): N-Desmethyl Tamoxifen $(2.05 \mathrm{~g}, 5.20 \mathrm{mmol})$ and glycol $2(2.72 \mathrm{~g}$, $7.80 \mathrm{mmol})$ were dissolved in anhydrous DMF $(60 \mathrm{~mL})$, under an atmosphere of argon gas. To this stirring mixture was added $\mathrm{K}_{2} \mathrm{CO}_{3}(7.19 \mathrm{~g}, 5.21 \mathrm{mmol})$ in one portion. The resulting mixture was left to stir at $85^{\circ} \mathrm{C}$ for 24 hours. After this time, DMF was evaporated under reduced pressure and the crude mixture was diluted by the addition of ethyl acetate. The excess $\mathrm{K}_{2} \mathrm{CO}_{3}$ was filtered off through a pad of celite eluted with $1: 12 \mathrm{MeOH} / \mathrm{CH}_{2} \mathrm{Cl}_{2}$ and the filtrate was then concentrated under reduced pressure. The resulting crude material was purified via silica gel flash column chromatography $\left(30: 70\right.$ acetone/ $\mathrm{CH}_{2} \mathrm{Cl}_{2} \rightarrow$ 5:95 $\mathrm{MeOH} / \mathrm{CH}_{2} \mathrm{Cl}_{2} \rightarrow$ 20:80 $\left.\mathrm{MeOH} / \mathrm{CH}_{2} \mathrm{Cl}_{2}\right)$ to give compound 3 as an oil $\left(1.54 \mathrm{mg}, 55 \%\right.$ yield, $\mathrm{R}_{\mathrm{f}}=0.25$ in 1:12 $\left.\mathrm{MeOH} / \mathrm{CH}_{2} \mathrm{Cl}_{2}\right)$. $\mathbf{H}$ NMR (500.13 MHz, $\left.\mathrm{CD}_{3} \mathrm{OD}\right): \delta=7.35-7.31(\mathrm{~m}, 2 \mathrm{H}, 2 \times \mathrm{ArH}), 7.27-7.21(\mathrm{~m}$, $3 \mathrm{H}, 3 \times \mathrm{Ar} \underline{\mathrm{H}}), 7.18-7.09(\mathrm{~m}, 5 \mathrm{H}, 5 \times \mathrm{Ar} \underline{\mathrm{H}}), 6.75(\operatorname{app~d}, \mathrm{J}=8.9 \mathrm{~Hz}, 2 \mathrm{H}, 2 \times \mathrm{Ar} \underline{\mathrm{H}}), 6.53(\operatorname{app~d}, \mathrm{J}$ $=8.9 \mathrm{~Hz}, 2 \mathrm{H}, 2 \times \mathrm{Ar} \underline{\mathrm{H}}), 3.95\left(\mathrm{t}, \mathrm{J}=5.9 \mathrm{~Hz}, 2 \mathrm{H}, \mathrm{OC}_{2}\right), 3.70-3.55(\mathrm{~m}, 14 \mathrm{H}), 3.40(\mathrm{~s}, 1 \mathrm{H}, \mathrm{OH})$, $2.79(\mathrm{t}, \mathrm{J}=5.9 \mathrm{~Hz}, 2 \mathrm{H}), 2.67(\mathrm{t}, \mathrm{J}=5.9 \mathrm{~Hz}, 2 \mathrm{H}), 2.44\left(\mathrm{q}, \mathrm{J}=7.4 \mathrm{~Hz}, 2 \mathrm{H}, \mathrm{CH}_{2} \mathrm{CH}_{3}\right), 2.34(\mathrm{~s}, 3 \mathrm{H}$, $\left.\mathrm{NC}_{\underline{H}_{3}}\right), 0.93\left(\mathrm{t}, \mathrm{J}=7.4 \mathrm{~Hz}, 3 \mathrm{H}, \mathrm{CH}_{2} \underline{\mathrm{CH}}_{3}\right)$; ${ }^{1} \mathbf{C ~ N M R}\left(125.77 \mathrm{MHz}, \mathrm{CD}_{3} \mathrm{OD}\right): \delta=156.8,143.9$, 142.5, 141.4, 138.3, $131.9(\mathrm{Ar} \underline{\mathrm{CH}}), 129.8(\mathrm{ArCH}), 129.5(\mathrm{Ar} \underline{\mathrm{CH}}), 128.2(\mathrm{ArCH}), 127.9(\mathrm{ArCH})$, $126.6(\mathrm{Ar} \underline{\mathrm{CH}}), 126.1(\mathrm{Ar} \underline{\mathrm{CH}}), 113.5(\mathrm{Ar} \underline{\mathrm{CH}}), 73.0,72.7\left(\underline{\mathrm{CH}}_{2}\right), 70.7\left(\underline{\mathrm{CH}}_{2}\right), 70.6\left(\underline{\mathrm{CH}}_{2}\right), 70.4,69.3$ $\left(\underline{\mathrm{CH}_{2}}\right), 65.7\left(\underline{\mathrm{CH}}_{2}\right), 61.8\left(\underline{\mathrm{CH}}_{2}\right), 61.7\left(\underline{\mathrm{CH}}_{2}\right), 57.1\left(\underline{\mathrm{CH}}_{2}\right), 56.6\left(\underline{\mathrm{CH}}_{2}\right), 43.4\left(\underline{\mathrm{CH}}_{3}\right), 29.1\left(\underline{\mathrm{CH}}_{2}\right), 13.7$

$\left(\mathrm{CH}_{3}\right)$; HRMS (ESI): calc. $\mathrm{C}_{33} \mathrm{H}_{44} \mathrm{NO}_{5}[\mathrm{M}+\mathrm{H}]+534.3219$, found 534.3233. IR (neat): $\mathrm{v}=3415$ $(\mathrm{OH}), 2870\left(-\mathrm{O}-\mathrm{CH}_{2}\right), 1605$ (-C=C-), 1573, 1507, 1461, 1443, $1349 \mathrm{~cm}^{-1}$.

Synthesis of (E)-1-(4-(1,2-diphenylbut-1-en-1-yl)phenoxy)-3-methyl-6,9,12-trioxa-3azatetradecan-14-yl 4-methylbenzenesulfonate (4): Compound $\mathbf{3}$ (570 mg, $1.07 \mathrm{mmol}$ ) was dissolved in anhydrous $\mathrm{CH}_{2} \mathrm{Cl}_{2}(15 \mathrm{~mL})$ and the mixture was cooled to $0^{\circ} \mathrm{C}$ in an ice bath, under an atmosphere of argon gas. To this cooled mixture was added freshly prepared $\mathrm{Ag}_{2} \mathrm{O}(588 \mathrm{mg}$, $2.54 \mathrm{mmol})$, KI (294 mg, $1.77 \mathrm{mmol})$, and TsCl (305 mg, $1.60 \mathrm{mmol})$. The resulting mixture was left to stir at $0^{\circ} \mathrm{C}$ for 30 minutes, then at reflux for 18 hours. After this time, the $\mathrm{Ag}_{2} \mathrm{O}$ was filtered off through a pad of celite eluted with $1: 12 \mathrm{MeOH} / \mathrm{CH}_{2} \mathrm{Cl}_{2}$. The filtrate was then concentrated under reduced pressure. The resulting crude material was subjected to silica gel column chromatography (40:60 acetone/ $\mathrm{CH}_{2} \mathrm{Cl}_{2} \rightarrow 5: 95 \mathrm{MeOH} / \mathrm{CH}_{2} \mathrm{Cl}_{2} \rightarrow 20: 80 \mathrm{MeOH} / \mathrm{CH}_{2} \mathrm{Cl}_{2}$ ) to give compound 4 as a colourless oil $\left(551 \mathrm{mg}, 75 \%\right.$ yield, $\mathrm{R}_{\mathrm{f}}=0.55$ in $\left.1: 10 \mathrm{MeOH} / \mathrm{CH}_{2} \mathrm{Cl}_{2}\right) .{ }^{1} \mathbf{H}$ NMR (399.85 MHz, CD OD) $\delta=7.76$ (app d, J = 8.9 Hz, 2H, $2 \times$ ArH $)$, 7.40-7.29 (m, 4H, $4 \times$ $\operatorname{Ar} \underline{\mathrm{H}}), 7.27-7.18(\mathrm{~m}, 3 \mathrm{H}, 3 \times \operatorname{Ar} \underline{\mathrm{H}}), 7.14-7.03(\mathrm{~m}, 5 \mathrm{H}, 5 \times \mathrm{Ar} \underline{\mathrm{H}}), 6.76(\operatorname{app~d}, \mathrm{J}=8.9 \mathrm{~Hz}, 2 \mathrm{H}, 2 \times$ $\operatorname{Ar} \underline{\mathrm{H}}), 6.54(\operatorname{app~d}, \mathrm{J}=8.9 \mathrm{~Hz}, 2 \mathrm{H}, 2 \times \mathrm{Ar} \underline{\mathrm{H}}), 4.10-4.06\left(\mathrm{~m}, 2 \mathrm{H}, \mathrm{OC}_{2}\right), 3.93(\mathrm{t}, \mathrm{J}=5.6 \mathrm{~Hz}, 2 \mathrm{H})$, $3.61-3.42(\mathrm{~m}, 12 \mathrm{H}), 2.82(\mathrm{t}, \mathrm{J}=5.6 \mathrm{~Hz}, 2 \mathrm{H}), 2.69(\mathrm{t}, \mathrm{J}=5.6 \mathrm{~Hz}, 2 \mathrm{H}), 2.43(\mathrm{q}, \mathrm{J}=7.4 \mathrm{~Hz}, 2 \mathrm{H}$, $\left.\mathrm{CH}_{2} \mathrm{CH}_{3}\right), 2.40\left(\mathrm{~s}, 3 \mathrm{H}, \mathrm{CH}_{3}\right), 2.34\left(\mathrm{~s}, 3 \mathrm{H}, \mathrm{NCH}_{3}\right), 0.89\left(\mathrm{t}, \mathrm{J}=7.4 \mathrm{~Hz}, 3 \mathrm{H}, \mathrm{CH}_{2} \mathrm{CH}_{3}\right) ;{ }^{3} \mathbf{C ~ N M R}$ $\left(100.54 \mathrm{MHz}, \mathrm{CD}_{3} \mathrm{OD}\right): \delta=158.0,146.4,145.0,143.6,142.6,139.8,136.9,134.4,132.9$ $(\mathrm{Ar} \underline{\mathrm{CH}}), 131.0(\mathrm{Ar} \underline{\mathrm{CH}}), 130.8(\mathrm{Ar} \underline{\mathrm{CH}}), 130.4(\mathrm{Ar} \underline{\mathrm{CH}}), 129.2(\mathrm{Ar} \underline{\mathrm{CH}}), 129.0(\mathrm{Ar} \underline{\mathrm{C}}), 128.9$

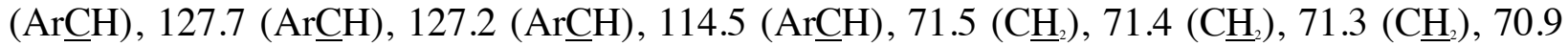
$\left(\mathrm{C}_{2}\right), 69.6\left(\underline{\mathrm{CH}}_{2}\right), 69.5\left(\underline{\mathrm{CH}}_{2}\right), 66.3\left(\mathrm{CH}_{2}\right), 57.8\left(\mathrm{C}_{2}\right), 57.2\left(\underline{\mathrm{CH}}_{2}\right), 43.5\left(\underline{\mathrm{CH}}_{3}\right), 29.9\left(\mathrm{C}_{2}\right), 21.7$ $\left(\mathrm{CH}_{3}\right), 13.9\left(\mathrm{CH}_{3}\right) ;$ HRMS (ESI): calc. $\mathrm{C}_{40} \mathrm{H}_{50} \mathrm{NO}_{7} \mathrm{~S}[\mathrm{M}+\mathrm{H}]^{+} 688.3308$, found 688.3320 . 
Synthesis of (E)-S-(1-(4-(1,2-diphenylbut-1-en-1-yl)phenoxy)-3-methyl-6,9,12-trioxa-3azatetradecan-14-yl) ethanethioate (5): Compound 4 (98 $\mathrm{mg}, 0.14 \mathrm{mmol})$ was dissolved in anhydrous THF ( $2 \mathrm{~mL})$, under an atmosphere of argon gas. To this mixture was added KSAc (81 $\mathrm{mg}, 0.71 \mathrm{mmol})$. The resulting mixture was heated at reflux for 16 hours. After this time, the residue was diluted with EtOAc and decoloursing charcoal was added. The decoloursing charcoal was filtered off through a pad of celite eluted with 1:12 $\mathrm{MeOH} / \mathrm{CH}_{2} \mathrm{Cl}_{2}$ and the filtrate was then concentrated under reduced pressure. The resulting crude material was purified via silica gel flash column chromatography (40:60 acetone/ $\mathrm{CH}_{2} \mathrm{Cl}_{2} \rightarrow 5: 95 \mathrm{MeOH} / \mathrm{CH}_{2} \mathrm{Cl}_{2}$ ) to give thioacetate 5 as an oil (38 mg, $46 \%$ yield, $\mathrm{R}_{t}=0.71$ in $\left.1: 12 \mathrm{MeOH} / \mathrm{CH}_{2} \mathrm{Cl}_{2}\right)$. ${ }^{1} \mathbf{H}$ NMR (399.85 $\left.\mathrm{MHz}, \mathrm{CD}_{3} \mathrm{OD}\right): \delta=7.36-7.28(\mathrm{~m}, 2 \mathrm{H}, 2 \times \mathrm{Ar} \underline{\mathrm{H}}), 7.27-7.19(\mathrm{~m}, 3 \mathrm{H}, 3 \times \mathrm{Ar} \underline{\mathrm{H}}), 7.19-7.04(\mathrm{~m}, 5 \mathrm{H}$, $5 \times \operatorname{Ar} \underline{\mathrm{H}}), 6.75(\operatorname{app~d}, \mathrm{J}=8.9 \mathrm{~Hz}, 2 \mathrm{H}, 2 \times \mathrm{Ar} \underline{\mathrm{H}}), 6.59(\operatorname{app~d}, \mathrm{J}=8.9 \mathrm{~Hz}, 2 \mathrm{H}, 2 \times \mathrm{Ar} \underline{\mathrm{H}}), 3.93(\mathrm{t}, \mathrm{J}$ $=5.9 \mathrm{~Hz}, 2 \mathrm{H}), 3.69-3.46(\mathrm{~m}, 12 \mathrm{H}), 3.06(\mathrm{t}, \mathrm{J}=6.5 \mathrm{~Hz}, 2 \mathrm{H}), 2.80(\mathrm{t}, \mathrm{J}=5.9 \mathrm{~Hz}, 2 \mathrm{H}), 2.68(\mathrm{t}, \mathrm{J}=$ $5.9 \mathrm{~Hz}, 2 \mathrm{H}), 2.44(\mathrm{q}, \mathrm{J}=7.4 \mathrm{~Hz}, 2 \mathrm{H}), 2.35\left(\mathrm{~s}, 3 \mathrm{H}, \mathrm{CH}_{3}\right), 2.30\left(\mathrm{~s}, 3 \mathrm{H}, \mathrm{C}_{\mathrm{H}}\right), 0.91(\mathrm{t}, \mathrm{J}=7.4 \mathrm{~Hz}, 3 \mathrm{H}$, $\left.\mathrm{C}_{3}\right)$; ${ }^{1} \mathrm{C}$ NMR (100.54 MHz, $\left.\mathrm{CD}_{3} \mathrm{OD}\right): \delta=195.4,156.7,143.8,142.4,141.3,138.3,135.5$, $131.8(\mathrm{Ar} \underline{\mathrm{CH}}), 129.7(\mathrm{Ar} \underline{\mathrm{CH}}), 129.5(\mathrm{Ar} \underline{\mathrm{CH}}), 128.1(\mathrm{Ar} \underline{\mathrm{CH}}), 127.9(\mathrm{Ar} \underline{\mathrm{CH}}), 126.5(\mathrm{Ar} \underline{\mathrm{CH}}), 126.0$ $(\mathrm{Ar} \underline{\mathrm{CH}}), 113.4(\mathrm{Ar} \underline{\mathrm{CH}}), 70.6\left(\underline{\mathrm{CH}}_{2}\right), 70.5\left(\underline{\mathrm{CH}}_{2}\right), 70.4\left(\underline{\mathrm{CH}}_{2}\right), 70.3\left(\underline{\mathrm{CH}}_{2}\right), 69.8\left(\underline{\mathrm{CH}}_{2}\right), 69.2\left(\underline{\mathrm{CH}}_{2}\right)$, $65.7\left(\underline{\mathrm{CH}}_{2}\right), 57.1\left(\underline{\mathrm{CH}}_{2}\right), 56.5\left(\underline{\mathrm{CH}}_{2}\right), 43.4\left(\underline{\mathrm{CH}}_{3}\right), 30.6\left(\underline{\mathrm{CH}}_{3}\right), 29.0\left(\underline{\mathrm{CH}}_{2}\right), 28.9\left(\underline{\mathrm{CH}}_{2}\right), 13.6\left(\underline{\mathrm{CH}}_{3}\right)$; HRMS (ESI): calc. $\mathrm{C}_{33} \mathrm{H}_{40} \mathrm{NO}_{5} \mathrm{~S}[\mathrm{M}+\mathrm{H}]+592.3097$, found 592.3085.

Synthesis of (E)-1-(4-(1,2-diphenylbut-1-en-1-yl)phenoxy)-3-methyl-6,9,12-trioxa-3azatetradecane-14-thiol (6): Thioacetate $5(50 \mathrm{mg}, 0.084 \mathrm{mmol})$ was dissolved in degassed EtOH $(400 \mu \mathrm{L})$, under an atmosphere of argon gas. To this mixture was added a solution of $\mathrm{NaOH}$ in degassed $\mathrm{H}_{2} \mathrm{O}(200 \mu \mathrm{L}, 7 \mathrm{M})$. The resulting mixture was heated at reflux for $2 \mathrm{hr}$. After this time, the reaction mixture was diluted with dichloromethane $(4 \mathrm{~mL})$. The layers were separated and the organic layer was washed with brine $(1 \mathrm{~mL})$. The organic layer was then filtered through a small plug of silica eluted with 5:95 $\mathrm{MeOH} / \mathrm{CH}_{2} \mathrm{Cl}_{2}$ and concentrated to give thiol $\mathbf{6}$ as an oil (33 mg, 71\% yield, 0.72 in 1:12 $\left.\mathrm{MeOH} / \mathrm{CH}_{2} \mathrm{Cl}_{2}\right)$. $\mathbf{H} \mathbf{~ N M R}\left(600.13 \mathrm{MHz}, \mathrm{CDCl}_{3}\right)$ : $\delta=7.36-7.32(\mathrm{~m}, 2 \mathrm{H}, 2 \times \mathrm{Ar} \underline{\mathrm{H}}), 7.28-7.22(\mathrm{~m}, 3 \mathrm{H}, 3 \times \mathrm{Ar} \underline{\mathrm{H}}), 7.19-7.15(\mathrm{~m}, 2 \mathrm{H}, 2 \times \mathrm{Ar} \underline{\mathrm{H}}), 7.13-$ $7.10(\mathrm{~m}, 3 \mathrm{H}, 3 \times \mathrm{Ar} \underline{\mathrm{H}}), 6.76(\operatorname{app~d}, \mathrm{J}=8.9 \mathrm{~Hz}, 2 \mathrm{H}, 2 \times \mathrm{Ar} \underline{\mathrm{H}}), 6.53(\operatorname{app~d}, \mathrm{J}=8.9 \mathrm{~Hz}, 2 \mathrm{H}, 2 \times$ $\operatorname{Ar} \underline{\mathrm{H}}), 3.97-3.93\left(\mathrm{~m}, 2 \mathrm{H}, \mathrm{OCH}_{2}\right), 3.72-3.56(\mathrm{~m}, 12 \mathrm{H}), 2.86(\mathrm{t}, \mathrm{J}=6.72 \mathrm{~Hz}, 2 \mathrm{H}), 2.83-2.78(\mathrm{~m}$, $2 \mathrm{H}), 2.72-2.66(\mathrm{~m}, 2 \mathrm{H}), 2.45\left(\mathrm{q}, \mathrm{J}=7.42 \mathrm{~Hz}, 2 \mathrm{H}, \mathrm{CH}_{2} \mathrm{CH}_{3}\right), 2.36\left(\mathrm{~s}, 3 \mathrm{H}, \mathrm{NCH}_{3}\right), 0.92(\mathrm{~T}, \mathrm{~J}=7.42$ $\left.\mathrm{Hz}, 3 \mathrm{H}, \mathrm{CH}_{2} \mathrm{CH}_{3}\right) ;{ }^{1} \mathbf{C}$ NMR $\left(100.54 \mathrm{MHz} \mathrm{CDCl}_{3}\right): \delta=156.8,143.9,142.5,141.4,138.3,135.6$, $131.9(\mathrm{ArCH}), 129.8(\mathrm{ArCH}), 129.5(\mathrm{ArCH}), 128.1(\mathrm{ArCH}), 127.9(\mathrm{Ar} \underline{\mathrm{CH}}), 126.6(\mathrm{Ar} \underline{\mathrm{CH}}), 126.0$ (Ar $\underline{\mathrm{CH}}), 113.4(\mathrm{Ar} \underline{\mathrm{CH}}), 77.4,70.7\left(\underline{\mathrm{CH}}_{2}\right), 70.6\left(\underline{\mathrm{CH}}_{2}\right), 70.5\left(\underline{\mathrm{CH}}_{2}\right), 70.4\left(\underline{\mathrm{CH}}_{2}\right), 69.5\left(\underline{\mathrm{CH}}_{2}\right), 65.9$ $\left(\underline{\mathrm{CH}}_{2}\right), 57.2\left(\underline{\mathrm{CH}}_{2}\right), 56.7\left(\underline{\mathrm{CH}}_{2}\right), 43.5\left(\underline{\mathrm{CH}}_{3}\right), 30.1\left(\underline{\mathrm{CH}}_{2}\right), 29.1\left(\underline{\mathrm{CH}}_{2}\right), 13.7\left(\underline{\mathrm{CH}}_{3}\right) ; \mathbf{H R M S}(\mathrm{ESI})$ : calc. $\mathrm{C}_{33} \mathrm{H}_{44} \mathrm{NO}_{4} \mathrm{~S}[\mathrm{M}+\mathrm{H}]^{+} 550.2991$, found 550.3018 .

Conjugation of (E)-1-(4-(1,2-diphenylbut-1-en-1-yl)phenoxy)-3-methyl-6,9,12-trioxa-3azatetradecane-14-thiol with BIODPY fluorophore (7): Commercially available BODIPY® FL N-(2-aminoethyl)maleimide (Life Technology, $5 \mathrm{mg}$ ) was dissolved in dry DMSO $(2 \times 0.25$ $\mathrm{mL})$ and transferred to a flask containing thiol $\mathbf{6}(10 \mathrm{mg})$. The resulting mixture was left to stir at room temperature under an atmosphere of argon gas, overnight, in the dark. After this time, the reaction mixture was diluted with methanol, separated into two batches and then freeze-dried using a lyophilizer to give a crude orange solid. Each batch of dry lyophised sample was then dissolved in methanol ( $1 \mathrm{~mL})$ and separated by HPLC. The column was eluted at $4 \mathrm{~mL} / \mathrm{min}$ with an isocratic mobile phase consisting of $65 \%$ acetonitrile: $35 \%$ of $0.1 \%$ TFA/water over $40 \mathrm{~min}$. 
UV absorbance was measured at wavelengths of 280, 488, and $508 \mathrm{~nm}$. Fractions were collected every minute for $40 \mathrm{~min}$. A sample of 7 eluted between 15 and $20 \mathrm{~min}$. The fractions containing the appropriate product were then concentrated under reduced pressure to remove acetonitrile and then freeze-dried using a lyophilizer to give 7 as an orange solid (3 mg). $\mathbf{H ~ N M R ~ ( 6 0 0 . 1 3 ~}$ $\left.\mathrm{MHz}, \mathrm{CD}_{3} \mathrm{OD}\right): \delta=7.41(\mathrm{~s}, 1 \mathrm{H}, \mathrm{C}=\mathrm{C} \underline{\mathrm{H}}), 7.37-7.33(\mathrm{~m}, 2 \mathrm{H}, 2 \times \operatorname{Ar} \underline{\mathrm{H}}), 7.29-7.25(\mathrm{~m}, 1 \mathrm{H}, 1 \times$ $\operatorname{Ar} \underline{\mathrm{H}}), 7.22-7.20(\mathrm{~m}, 2 \mathrm{H}, 2 \times \mathrm{Ar} \underline{\mathrm{H}}), 7.17-7.07$ (m, 5H, $5 \times \mathrm{Ar} \underline{\mathrm{H}}), 6.99(\operatorname{app~d}, \mathrm{J}=4.14 \mathrm{~Hz}, 1 \mathrm{H}$, $\mathrm{C}=\mathrm{C} \underline{\mathrm{H}}), 6.83(\operatorname{app~d}, \mathrm{J}=8.9 \mathrm{~Hz}, 2 \mathrm{H}, 2 \times \mathrm{Ar} \underline{\mathrm{H}}), 6.65(\operatorname{app~d}, \mathrm{J}=8.9 \mathrm{~Hz}, 2 \mathrm{H}, 2 \times \mathrm{Ar} \underline{\mathrm{H}}), 6.30$ (app $\mathrm{d}, \mathrm{J}=4.14 \mathrm{~Hz}, 1 \mathrm{H}, \mathrm{C}=\mathrm{CH}), 6.20(\mathrm{~s}, 1 \mathrm{H}, \mathrm{C}=\mathrm{C} \underline{\mathrm{H}}), 4.24-4.22(\mathrm{~m}, 2 \mathrm{H}), 3.84-3.80(\mathrm{~m}, 2 \mathrm{H}), 3.70-$ $3.55(\mathrm{~m}, 12 \mathrm{H}), 3.05-2.99(\mathrm{~m}, 2 \mathrm{H}), 2.97\left(\mathrm{~s}, 3 \mathrm{H}, \mathrm{CH}_{3}\right), 2.86-2.80(\mathrm{~m}, 2 \mathrm{H}), 2.55-2.52(\mathrm{~m}, 2 \mathrm{H}), 2.49$

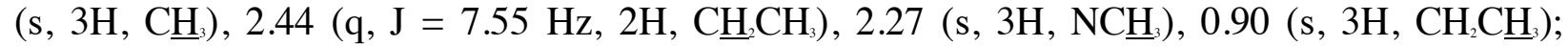
HRMS (ESI): calc. $\mathrm{C}_{47} \mathrm{H}_{55} \mathrm{BF}_{2} \mathrm{~N}_{3} \mathrm{O}_{5} \mathrm{~S}[\mathrm{M}+\mathrm{H}]+822.3924$, found 822.3921 .

26. Olofson, R. A.; Martz, J. T.; Senet, J. P.; Piteau, M.; Malfroot, T. J. Org. Chem. 1984, 49, 2081-2082.

27. Bouzide, A.; Sauvé, G. Org. Lett. 2002, 4, 2329-2332.

28. Cell Culture and bioconjugate incubation: The ER positive MCF7 and ER negative MDA231 breast cancer cells were cultured in RPMI media plus 10\% fetal bovine serum (Life Technologies) and grown at $37^{\circ} \mathrm{C}$ in a humidifying incubator. Cells were sub-cultured onto glass coverslips in sterile six-well plates at a concentration of $4 \times 10^{\text {s }}$ cells $/ \mathrm{mL}$ for labeling experiments. Sub-confluent cultures were incubated for one hour in bodipy-labelled tamoxifen (7) diluted in culture media to $10 \mu \mathrm{g} / \mathrm{mL}$ (low conc. TAM) or $100 \mu \mathrm{g} / \mathrm{mL}$ (high conc. TAM), then rinsed and replaced into normal growth media immediately prior to imaging. Live stained cells were mounted under buffered saline and visualized on a Nikon A1Si fluorescent confocal microscope. Sequential fields were captured in the z-plane to confirm internal cellular localization. 